\title{
Mesenteric lymph reperfusion after superior mesenteric artery occlusion shock exacerbates endotoxin translocation in brain ${ }^{1}$
}

\author{
Zi-gang Zhao', Li-na Yang ${ }^{\mathrm{II}}$, Yong-quan Zhao ${ }^{\mathrm{III}}$, Chun-yu Niu ${ }^{\mathrm{IV}}$ \\ DOI: http://dx.doi.org/10.1590/S0102-86502014000600002
}

${ }^{\mathrm{I}}$ Master, Full Professor, Institute of Microcirculation, Hebei North University, Zhangjiakou, China. Acquisition of data, manuscript writing, critical revision.

IIMaster, Physician, Institute of Microcirculation, Hebei North University, Zhangjiakou, China. Acquisition of data.

"IIBachelor, Chief physician, Full Professor, Institute of Microcirculation, Hebei North University, Zhangjiakou, China. Acquisition of data.

${ }^{\text {IV }} \mathrm{PhD}$, Full Professor, Institute of Microcirculation, Hebei North University, Zhangjiakou, China. Design of the study, critical revision.

\begin{abstract}
PURPOSE: To determine the role of mesenteric lymph reperfusion (MLR) on endotoxin translocation in brain to discuss the mechanism of brain injury subjected to superior mesenteric artery occlusion (SMAO) shock.

METHODS: Twenty-four rats were randomly assigned to MLR, SMAO, MLR+SMAO and sham groups. MLR was performed by clamping the mesenteric lymph duct (MLD) for $1 \mathrm{~h}$ and then allowing reperfusion for $2 \mathrm{~h}$ in the MLR group; SMAO involved clamping the superior mesenteric artery (SMA) for $1 \mathrm{~h}$, followed by reperfusion for $2 \mathrm{~h}$ in the SMAO group; occlusion of both the SMA and MLD for $1 \mathrm{~h}$ was followed by reperfusion for $2 \mathrm{~h}$ in the MLR+SMAO group rats.

RESULTS: SMAO shock induced severe increased levels of the endotoxin, lipopolysaccharide receptor, lipopolysaccharide-binding protein, intercellular adhesion molecule-1 and tumor necrosis factor- $\alpha$. Concurrently, MLR after SMAO shock further aggravates these deleterious effects.
\end{abstract}

CONCLUSION: Mesenteric lymph reperfusion exacerbated the endotoxin translocation in brain; thereby increased inflammatory response occurred, suggesting that the intestinal lymph pathway plays an important role in the brain injury after superior mesenteric artery occlusion shock.

Key words: Shock. Lymph. Reperfusion Injury. Mesenteric Artery, Superior. Brain Injuries. Endotoxins. Rats. 


\section{Introduction}

Superior mesenteric artery occlusion (SMAO) shock is a severe pathological process resulting from ischemia/ reperfusion $(\mathrm{I} / \mathrm{R})$ injury. Intestinal barrier dysfunction due to intestinal $\mathrm{I} / \mathrm{R}$ may result in bacterial/endotoxin translocation (BET) and the release of inflammatory mediators/cytokines that cause dysfunction and structural damage in remote organs, thereby leading to the induction of multiple organ dysfunction syndrome (MODS) or even multiple organ failure $(\mathrm{MOF})^{1-3}$, which is a major cause of death. It has been demonstrated that ligation of the mesenteric lymph duct (MLD) may alleviate the dysfunction and morphologic changes in multiple organs in rats with two-hit of hemorrhage and lipopolysaccharide injection ${ }^{4}$, and hemorrhagic shock ${ }^{5-8}$; similarly, the mesenteric lymph significantly contribute to the development of irreversible shock after $\mathrm{SMAO}^{9}$. In conclusion, the intestinal lymph pathway plays an important role in the pathological process ${ }^{10,11}$.

Because the MLD has a thin and transparent wall and is adjacent to the superior mesenteric artery (SMA), they can inadvertently be ligated together during the SMAO shock establishment. Thus, the mesenteric lymph after occlusion will return to lymphatic system, and the role of mesenteric lymph reperfusion (MLR) in the pathogenesis of SMAO shock is notable. Previous studies showed that MLR could exacerbate SMAO shock-induced brain damage and decrease the excitatory neurotransmitter level ${ }^{12,13}$. However, the mechanism needs further research. Several studies have suggested that enterogenous BET was an important mechanism of organ injuries following hemorrhagic shock ${ }^{14,15}$. Meanwhile, the intestinal lymph pathway has been suggested to be involved in intestinal BET following intestinal ischemia/reperfusion ${ }^{16}$. Therefore, in the present study, we examined the role of MLR after SMAO on endotoxin translocation, to investigate the mechanism by which SMAO shock induces brain injury.

\section{Methods}

All animal procedures were reviewed and approved by the Institutional Animal Care and Use Committee of Hebei North University and conformed to National Institutes of Health guidelines. All efforts were made to minimize suffering of animals.

Twenty-four adult male Wistar rats weighing 280-350 g (provide by the Laboratory Animal Breeding Center of the Chinese Academy of Medical Sciences) were randomly divided into four groups ( $\mathrm{n}=6$ per group): MLR, SMAO, MLR+SMAO and sham groups. Before experimentation, the rats were fasted for $12 \mathrm{~h}$, but were allowed free access to water.

The animals were anesthetized with pentobarbital sodium $(50 \mathrm{mg} / \mathrm{kg})$. Under strict aseptic conditions, a $5-\mathrm{cm}$ midline laparotomy was performed. The superior mesenteric artery (SMA) was located by deflecting the loops of the intestine to the left with moist gauze swabs and separated from the accompanying mesenteric lymphatic trunk. In the SMAO group rats, the SMA was occluded for $1 \mathrm{~h}$ by placing an atraumatic haemostatic clamp close to its origin in the aorta. This was followed by reperfusion for $2 \mathrm{~h}$. In the MLR group rats, the MLD was peeled away from the surrounding adipose and connective tissue and clamped for $1 \mathrm{~h}$ with an atraumatic haemostatic clamp. This was followed by reperfusion for $2 \mathrm{~h}$. In the MLR+SMAO group rats, occlusion of both the SMA and the MLD for $1 \mathrm{~h}$ was followed by reperfusion for $2 \mathrm{~h}$. The sham group rats underwent laparotomy, but the SMA and MLD were not occluded. Figure 1 shows the anatomic distribution of the MLD and SMA, and the experimental methods used in each group.
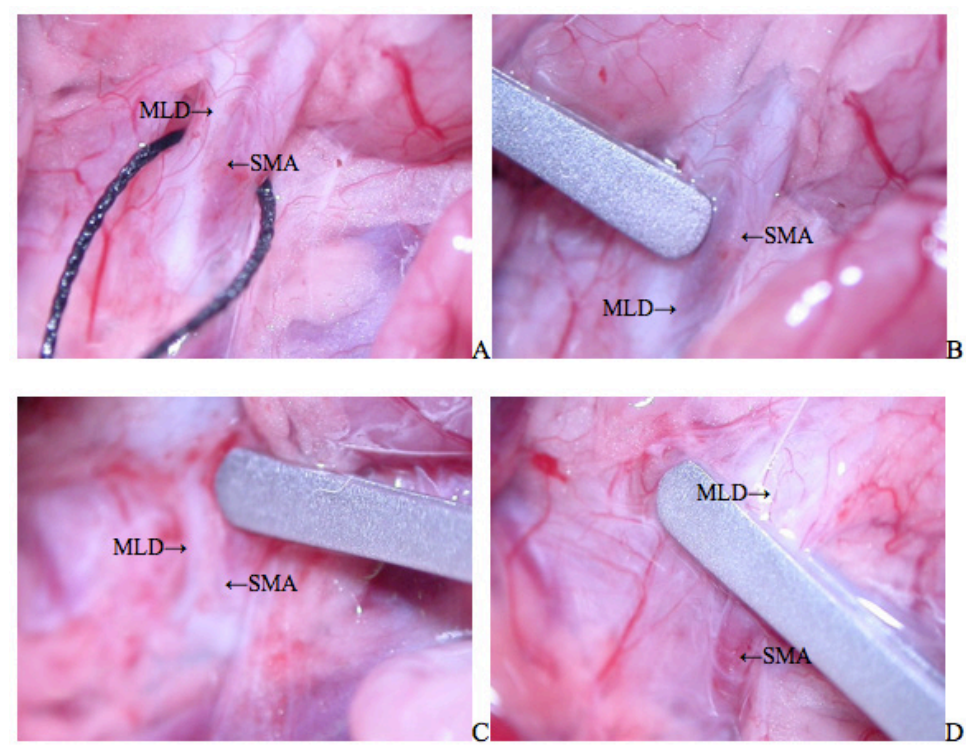

FIGURE 1 - The experimental methods used in each group. During the operation, the superior mesenteric artery (SMA) was separated from the mesenteric lymph duct (MLD) (i.e., the mesenteric lymphatic trunk). The SMA and MLD were not occluded in the sham group rats (see the A image); the MLD was clamped in the MLR group rats (see the B image); the SMA was occluded in the SMAO group rats (see the C image); and both the SMA and MLD were occluded in the MLR+SMAO group rats (see the D image). 


\section{Preparation of brain homogenate}

After reperfusion for $2 \mathrm{~h}$, under strict aseptic conditions, the brains were immediately removed for preparation of brain homogenate. A vertical incision was made at the combining site of the left and right hemispheres. Subsequently, the left brain tissue was mixed with nine times the amount of water, and was homogenized using glass homogenizer; then, the homogenate was sealed up to glass tube and stored in a $-80^{\circ} \mathrm{C}$ refrigerator (Thermo Electron, Waltham, MA) for the measurement of endotoxin levels. In accordance with the instructions for measuring bacterial endotoxin levels in the Chinese Pharmacopoeia, all appliances that came into contact with samples or water were treated by sterilization and were pyrogen-free, and the procedure were performed in a safety cabinet (Thermo Electron, Waltham, MA).

Moreover, the right brain tissue was mixed with five times the amount of $4^{\circ} \mathrm{C}$ normal saline to produce a $16.7 \%$ homogenate using a FJ-200 type high-speed dispersion machine (Shanghai Specimen and Model Factory, Shanghai, China) at low temperature. Next, the supernatant was collected by centrifugation for $10 \mathrm{~min}$ at $2.500 \mathrm{rpm}$ using a Labofuge 400R type lowtemperature centrifuge (Heraeus, Hanover, Germany), and was stored at $-80^{\circ} \mathrm{C}$ for the further measurement.

\section{Measurement of endotoxin levels}

Before the measurement of endotoxin levels in brain tissue, a standard curve was constructed. Standard endotoxin products (National Institute for the Control of Pharmaceutical and Biological Products, Beijing, China) were diluted to final concentrations of 2, 0.25 and $0.03125 \mathrm{EU} / \mathrm{ml}$ of bacterial endotoxin. Then, the standard solutions were added to a tachypleus amebocyte lysate (TAL) reaction tube. Each concentration was tested in duplicate using an ATi 320-06 32-well kinetic tube reader (Lab Kinetics Ltd., UK) that had been preheated to $37^{\circ} \mathrm{C}$. The detection wavelength was $405 \mathrm{~nm}$ and the preset threshold was $92 \%$. A standard curve was generated by linear regression analysis.

Brain homogenate was deproteinized through incubation at $70^{\circ} \mathrm{C}$ in a water bath for $10 \mathrm{~min}$ and then centrifuged at $3.000 \mathrm{rpm}$ for $10 \mathrm{~min}$. The supernatant was serial diluted in order to do the interference tests with the aim of measuring the recovery of the standard endotoxin products, and determining the optimum sample dilution from the values obtained. The optimum sample dilution was 1:80 according to the recovery test results and with reference to the Chinese Pharmacopoeia (2005 edition). The measurement of endotoxin levels was conducted using 80 -fold-diluted samples. Specimen plus standard endotoxin products $(0.25 \mathrm{EU} / \mathrm{ml})$ was used a positive control. Each sample was analyzed in duplicate. Data were collected and analyzed automatically using an endotoxin detector. The endotoxin content of each sample was calculated using the standard curve. Similarly, all appliances that came into contact with samples or reagents also were treated by sterilization and were pyrogen-free, and all procedures were performed in a safety cabinet.

\section{Enzyme-linked immunoadsorbent assay (ELISA)}

The contents of lipopolysaccharide receptor (CD14), lipopolysaccharide- binding protein (LBP), intercellular adhesion molecule-1 (ICAM-1), and tumor necrosis factor- $\alpha$ (TNF- $\alpha$ ) in brain homogenates were measured using rat ELISA kit (antibodies were purchased from R\&D Systems, USA), in accordance with the kit instructions after manufacturing a standard curve. And then, the total protein contents in brain homogenates were determined by Coomassie brilliant blue colorimetric method ${ }^{17}$ (Jiancheng Biotechnology Research Institute, Nanjing, China) for the standardization of these indices.

\section{Statistical analysis}

All data are expressed as the mean \pm SD and were analyzed using SPSS version 11.0 software. One-way analysis of variance was used to identify differences within groups, and paired $t$ tests were used to identify differences between groups. The KruskalWallis test was used to analyze data that was not suitable for oneway analysis of variance. A $P$ value of $<0.05$ was considered to be statistically significant.

\section{Results}

\section{Endotoxin levels in brain tissue}

The regression equation for the endotoxin standard curve was $\log \mathrm{T}=2.8369-0.2402 \log \mathrm{C}(r=-0.9837)$, where $\mathrm{T}$ is the response time and $\mathrm{C}$ is the endotoxin content (Figure $2 \mathrm{~A})$. Figure $2 \mathrm{~B}$ showed that there was no difference in ET level between the sham and MLR groups; the ET levels in the SMAO and SMAO+MLR groups were significantly increased than that of the sham and MLR groups; meanwhile, the ET level in the SMAP+MLR group was higher than that in the SMAO group. 

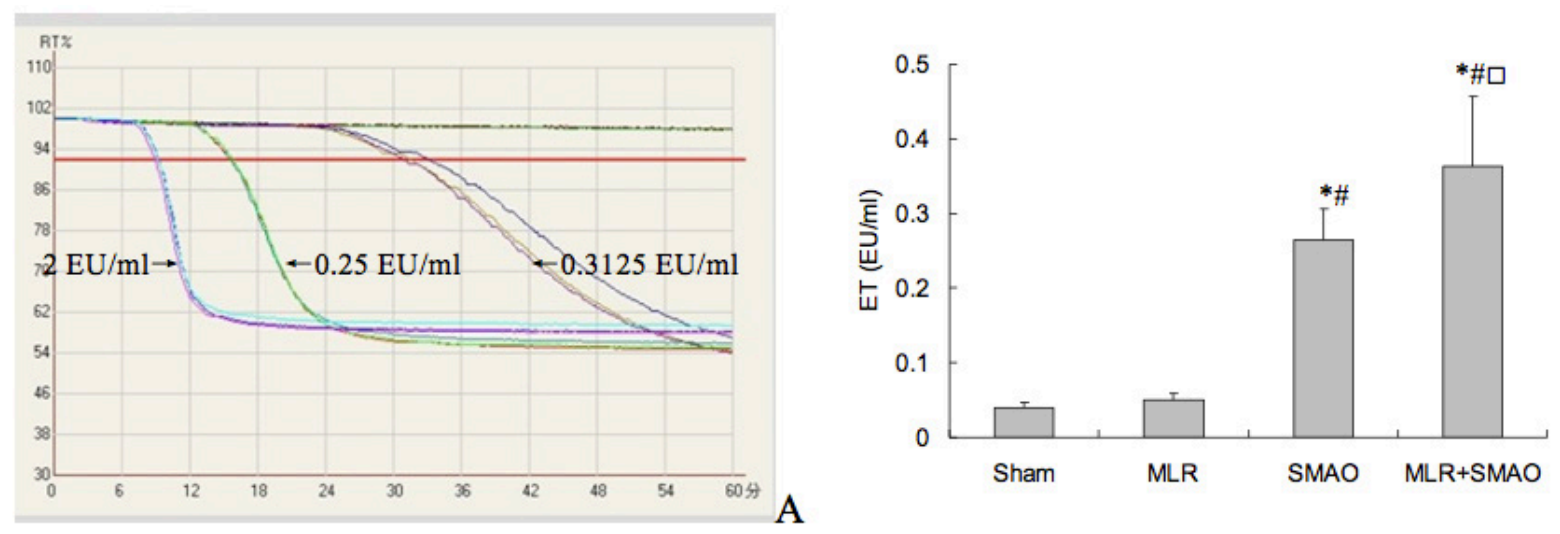

FIGURE 2 - Effect of mesenteric lymph reperfusion (MLR) after superior mesenteric artery occlusion (SMAO) shock on endotoxin (ET) level in brains of rats (mean $\pm \mathrm{SD}, n=6)$. A) Standard curve for endotoxin. B) ET levels in each group. ${ }^{*} P<0.05 v s$. the sham group; ${ }^{\#} P<0.05 v s$. the MLR group; ${ }^{\square} P<0.05$ vs. the SMAO group.

\section{CD14 and LBP levels in brain tissue}

As shown in Figure 3, the CD14 and LBP levels in brain tissue were comparable in the sham and MLR groups $(P>0.05)$; in contrast, these indices were significantly higher in the SMAO and MLR+SMAO groups than in the sham group, and were even increased in the MLR+SMAO group $(P<0.05)$.

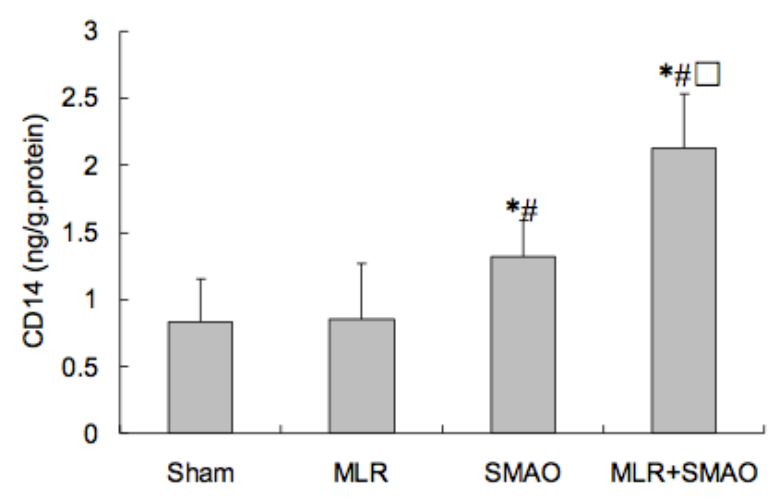

\section{ICAM-1 and TNF-á levels in brain tissue}

There were no significant differences between the sham and MLR groups in ICAM-1 and TNF- $\alpha$ used to assess inflammatory response induced by ET $(P<0.05)$. In comparison with the sham and MLR groups, the ICAM- 1 and TNF- $\alpha$ level in the brain tissue from rats in the SMAO and SMAO+MLR groups were significantly elevated. Moreover, the contents of ICAM-1 and TNF- $\alpha$ were significantly higher in the MLR+SMAO group than in the SMAO group (Figure 4).

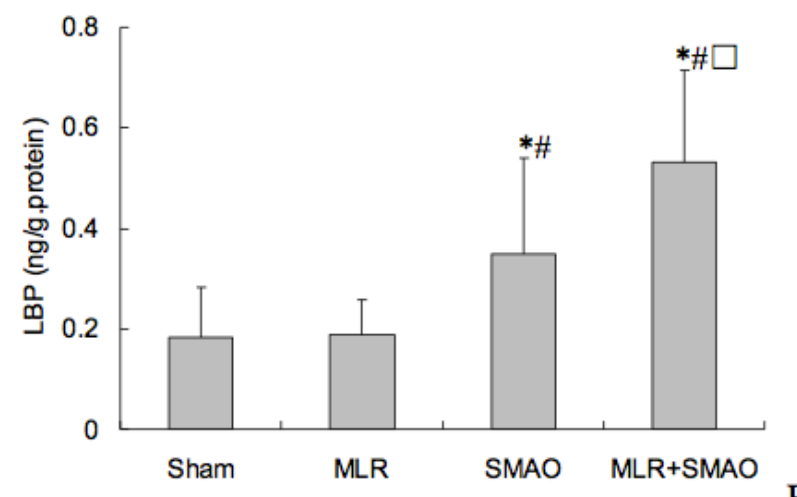

FIGURE 3 - Effect of mesenteric lymph reperfusion (MLR) after superior mesenteric artery occlusion (SMAO) shock on the lipopolysaccharide receptor (CD14) and lipopolysaccharide-binding protein (LBP) levels in brain of rats (mean $\pm \mathrm{SD}, n=6)$. A) CD14 level. B) LBP level. $* P<0.05 v s$. the sham group; ${ }^{\sharp} P<0.05$ vs. the MLR group; ${ }^{\square} P<0.05$ vs. the SMAO group.
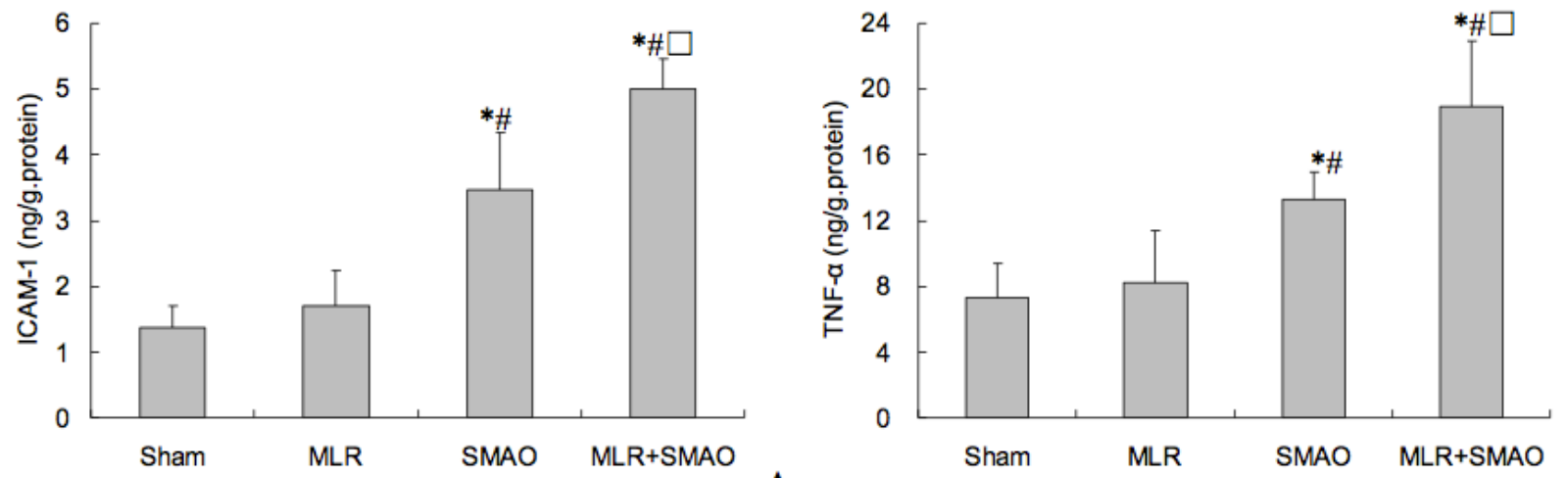

A

FIGURE 4 - Effect of mesenteric lymph reperfusion (MLR) after superior mesenteric artery occlusion (SMAO) shock on the indices related to inflammatory response in brain of rats $(\operatorname{mean} \pm \mathrm{SD}, n=6)$. A) Intercellular adhesion molecule-1 (ICAM-1) level. B) Tumor necrosis factor- $\alpha(\mathrm{TNF}-\alpha)$ level. ${ }^{*} P<0.05$ vs. the sham group; ${ }^{\sharp} P<0.05$ vs. the MLR group; ${ }^{\circ} P<0.05$ vs. the SMAO group. 


\section{Discussion}

The mononuclear macrophage system was activated by intestinal endotoxin translocation and releases a large amount of inflammatory mediators, which induces systemic inflammatory response syndrome and subsequent organ injury ${ }^{18}$. Therefore, in the present study, we investigated the effects of MLR after SMAO on endotoxin translocation for the first time (to the best of our knowledge). Our results show that simultaneous occlusion and reperfusion of the SMA and MLD exacerbates endotoxin level in brain tissue, as well as increasing levels of CD14, LBP, ICAM-1, and TNF- $\alpha$. These data suggest that the intestinal lymph pathway plays an important role in the pathogenesis of brain injury after SMAO shock.

The intestinal lymph pathway plays an important role in intestinal BET in severe pathological conditions, such as hemorrhagic and traumatic shock and acute pancreatitis ${ }^{10,11}$. The results of the present study show that the endotoxin level in brain tissue in rats following SMAO shock, which indicated that endotoxin translocation occurred. At the same time, the endotoxin content in SMAO+MLR group was significantly elevated than SMAO group, suggesting that MLR after SMAO increased the endotoxin translocation to brain tissue through the intestinal lymphatic pathway. Its mechanism might be related to mesenteric micro-lymphatic hyper-permeability following MLD occlusion and SMAO, as well as blood brain barrier injury. However, it needs further investigation.

Previous results showed that the LBP and membrane CD14 enhance the responses of both blood monocytes and tissue macrophages to endotoxin ${ }^{19}$. Therefore, inflammatory response of tissue injury mediated by endotoxin in an LBP- and CD14dependent manner, besides the endothelial cells injury directly induced by endotoxin. The present data show that SMAO shock increased the levels of LBP and CD14 in brain, and that MLR after SMAO exacerbated these changes, suggesting that LBP and CD14 are important contributors to the inflammatory response induced by endotoxin during the mechanism by which MLR aggravating the brain injury following SMAO shock.

It has been reported that increased ICAM-1 plays an important role in polymorphonuclear granulocyte adhesion to vascular endothelial cell subjected to hemorrhage and exposed to lipopolysaccharide, thereby causing inflammatory response and microcirculation disorders ${ }^{20,21}$. Our data show that reperfusion after ligation of the MLD exacerbates the ICAM-1 level in brain tissue following SMAO shock, which is related to endotoxin translocation. As a result, increased TNF- $\alpha$ appeared, suggesting
MLR enhancing the inflammatory cascade of brain through endotoxin translocation in rats subjected to SMAO shock.

In addition, we found that there were no significant differences in these parameters between the MLR and sham groups, indicating that the effect of MLR in physiological relevant condition is no harmful.

\section{Conclusion}

Mesenteric lymph reperfusion after superior mesenteric artery occlusion shock exacerbates endotoxin translocation, activates LBP/CD14 as endotoxin sensitizing system, and increases subsequent inflammatory response in brain tissue.

\section{References}

1. Moore FA. The role of the gastrointestinal tract in postinjury multiple organ failure. Am J Surg. 1999 Dec;178(6):449-53. doi: 10.1016/S0002-9610(99)00231-7.

2. Deitch EA. Gut-origin sepsis: evolution of a concept. Surgeon. 2012 Dec;10(6):350-6. doi: 10.1016/j.surge.2012.03.003.

3. Deitch EA, Feketeova E, Lu Q, Zaets S, Berezina TL, Machiedo GW, Hauser CJ, Livingston DH, Xu DZ. Resistance of the female, as opposed to the male, intestine to I/R-mediated injury is associated with increased resistance to gut-induced distant organ injury. Shock. 2008 Jan;29(1):78-83. doi: 10.1097/shk.0b013e31806ec26.

4. Niu CY, Li JC, Zhao ZG, Zhang J, Shao XH. Effect of intestinal lymphatic circulation blockage in two- hit rats. World J Gastroenterol. 2006 Sep;12(36):5805-12. doi: 10.3748/wjg.v12.i36.5805.

5. Reino DC, Pisarenko V, Palange D, Doucet D, Bonitz RP, Lu Q, Colorado I, Sheth SU, Chandler B, Kannan KB, Ramanathan M, $\mathrm{Xu}$ da Z, Deitch EA, Feinman R. Trauma hemorrhagic shockinduced lung injury involves a gut-lymph-induced TLR4 pathway in mice. PLoS One. 2011 Aug;6(8):e14829. doi: 10.1371/journal. pone.0014829.

6. Sambol J, Deitch EA, Takimoto K, Dosi G, Yatani A. Cellular basis of burn-induced cardiac dysfunction and prevention by mesenteric lymph duct ligation. J Surg Res. 2013 Aug;183(2):678-85. doi: 10.1016/j.jss.2013.01.065.

7. Niu CY, Zhao ZG, Ye YL, Hou YL, Zhang YP. Mesenteric lymph duct ligation against renal injury in rats after hemorrhagic shock. Ren Fail. 2010 Jun;32(5):584-91. doi: 10.3109/08860221003778031.

8. Caputo FJ, Rupani B, Watkins AC, Barlos D, Vega D, Senthil M, Deitch EA. Pancreatic duct ligation abrogates the trauma hemorrhage-induced gut barrier failure and the subsequent production of biologically active intestinal lymph. Shock. 2007 Oct;28(4):441-6. doi: 10.1097/shk.0b013e31804858f2

9. Badami CD, Senthil M, Caputo FJ, Rupani BJ, Doucet D, Pisarenko V, Xu DZ, Lu Q, Feinman R, Deitch EA. Mesenteric lymph duct ligation improves survival in a lethal shock model. Shock. 2008 Dec;30(6):680-5. doi: 10.1097/shk.0b013e318173edd1.

10. Deitch EA. Gut lymph and lymphatics: a source of factors leading to organ injury and dysfunction. Ann NYAcad Sci. 2010 Oct;1207(Suppl 1):E103-11. doi: 10.1111/j.1749-6632.2010.05713.x.

11. Cai B, Deitch EA, Ulloa L. Novel insights for systemic inflammation in sepsis and hemorrhage. Mediators Inflamm. 2010 Jun;2010:642462. doi: 10.1155/2010/642462.

12. Zhao ZG, Niu CY, Shang AM, Tian JM, Han R, Zhang CH, Zhang 
YP, Zhang J. Mesenteric lymph reperfusion may exacerbate brain injury in a rat model of superior mesenteric artery occlusion shock. Neural Regen Res. 2010 May;5(9):683-9. doi: 10.3969/j.issn.16735374.2010.09.008.

13. Niu CY, Zhao ZG, Shang AM, Tian JM, Zhang CH, Han R, Zhang YP, Zhang J. Influence of mesenteric lymph reperfusion on neurotransmitter expression in brain tissue of a superior mesenteric artery occlusion shock rat model. Neural Regen Res. 2010 Jun;5(11):859-63. doi: 10.3969/j.issn.1673-5374.2010.11.009.

14. Kao NR, Xenocostas A, Driman DK, Rui T, Huang W, Jiao X, Martin CM. Recombinant human erythropoietin improves gut barrier function in a hemorrhagic shock and resuscitation rat model. J Trauma. 2011 Nov;71(5 Suppl 1):S456-61. doi: 10.1097/ TA.0b013e318232e 782.

15. Filos KS, Panteli ES, Fligou F, Papamichail C, Papapostolou I, Zervoudakis G, Spiliopoulou I, Georgiou C. Clonidine pretreatment prevents hemorrhagic shock-induced endotoxemia and oxidative stress in the gut, liver, and lungs of the rat. Redox Rep. 2012 Nov; 17(6):246-51. doi: 10.1179/1351000212Y.0000000029.

16. Cavriani G, Domingos HV, Soares AL, Trezena AG, LigeiroOliveira AP, Oliveira-Filho RM, Sudo-Hayashi LS, Tavares de Lima W. Lymphatic system as a path underlying the spread of lung and gut injury after intestinal ischemia/reperfusion in rats. Shock. 2005 Apr;23(4):330-6. doi: 10.1097/shk.0b013e3181863080.

17. Bautista J, Corpas R, Ramos R, Cremades O, Gutiérrez JF, Alegre $\mathrm{S}$. Brain mitochondrial complex I inactivation by oxidative modification. Biochem Biophys Res Commun. 2000 Sep;275(3):8904. doi: 10.1006/bbrc.2000.3388.

18. Venet F, Davin F, Guignant C, Larue A, Cazalis MA, Darbon R, Allombert C, Mougin B, Malcus C, Poitevin-Later F, Lepape A, Monneret G. Early assessment of leukocyte alterations at diagnosis of septic shock. Shock. 2010 Oct;34(4):358-63. doi: 10.1097/ shk.0b013e3181dc0977.

19. Heumann D, Adachi Y, Le Roy D, Ohno N, Yadomae T, Glauser MP, Calandra T. Role of plasma, lipopolysaccharide- binding protein, and CD14 in response of mouse peritoneal exudate macrophages to endotoxin. Infect Immun. $2001 \mathrm{Jan} ; 69(1): 378-85$. doi: 10.1128/ IAI.69.1.378-385.2001.
20. Xu DZ, Lu Q, Adams CA, Issekutz AC, Deitch EA. Traumahemorrhagic shock-induced up-regulation of endothelial cell adhesion molecules is blunted by mesenteric lymph duct ligation. Crit Care Med. 2004 Mar;32(3):760-5. doi: 10.1097/01. ccm.0000114815.88622.9D.

21. Zhao ZG, Niu CY, Zhang LL, Zhang J, Han R, Zhang YP, Hou YL. Exogenous normal lymph alleviates lipopolysaccharide-induced acute kidney injury in rats. Ren Fail. 2013 Jul;35(6):806-11. doi: 10.3109/0886022X.2013.794680.

\section{Correspondence:}

Chunyu Niu

Institute of Microcirculation, Hebei North University

Zhangjiakou Hebei, 075000, China

Phone: (86)0313-4029168

ncylxf@126.com

lymphatics@126.com

Received: Jan 22, 2014

Review: March 20, 2014

Accepted: April 23, 2014

Conflict of interest: none

Financial sources: Key scientific and technological project of Hebei Province (09276101D-31, 11276103D-84) and Foundation of Hundred Innovative Talents in Universities of Hebei Province (CPRC047 and CPRCII026).

${ }^{1}$ Research performed at Institute of Microcirculation, Hebei North University, Zhangjiakou Hebei, China. 